\title{
Molecular and Enzymatic Features of Homoserine Dehydrogenase from Bacillus subtilis
}

\author{
Do Hyeon Kim ${ }^{1 \dagger}$, Quyet Thang Nguyen ${ }^{1,2 \dagger}$, Gyeong Soo $\mathrm{Ko}^{1 \dagger}$, and Jin Kuk Yang ${ }^{1 *}$ \\ 'Department of Chemistry, College of Natural Sciences, Soongsil University, Seoul 06978, Republic of Korea \\ ${ }^{2}$ Department of Information Communication, Materials, and Chemistry Convergence Technology, Soongsil \\ University, Seoul 06978, Republic of Korea
}

\begin{abstract}
Homoserine dehydrogenase (HSD) catalyzes the reversible conversion of L-aspartate-4semialdehyde to L-homoserine in the aspartate pathway for the biosynthesis of lysine, methionine, threonine, and isoleucine. HSD has attracted great attention for medical and industrial purposes due to its recognized application in the development of pesticides and is being utilized in the large scale production of L-lysine. In this study, HSD from Bacillus subtilis (BsHSD) was overexpressed in Escherichia coli and purified to homogeneity for biochemical characterization. We examined the enzymatic activity of BsHSD for L-homoserine oxidation and found that BsHSD exclusively prefers $\mathrm{NADP}^{+}$to $\mathrm{NAD}^{+}$and that its activity was maximal at $\mathrm{pH} 9.0$ and in the presence of $0.4 \mathrm{M} \mathrm{NaCl}$. By kinetic analysis, $K_{\mathrm{m}}$ values for L-homoserine and NADP ${ }^{+}$were found to be $35.08 \pm 2.91 \mathrm{mM}$ and $0.39 \pm 0.05 \mathrm{mM}$, respectively, and the $V_{\max }$ values were $2.72 \pm 0.06 \mu \mathrm{mol} / \mathrm{min}^{-1} \mathrm{mg}^{-1}$ and $2.79 \pm 0.11$ $\mu \mathrm{mol} / \mathrm{min}^{-1} \mathrm{mg}^{-1}$, respectively. The apparent molecular mass determined with size-exclusion chromatography indicated that BsHSD forms a tetramer, in contrast to the previously reported dimeric HSDs from other organisms. This novel oligomeric assembly can be attributed to the additional C-terminal ACT domain of BsHSD. Thermal denaturation monitoring by circular dichroism spectroscopy was used to determine its melting temperature, which was $54.8^{\circ} \mathrm{C}$. The molecular and biochemical features of BsHSD revealed in this study may lay the foundation for future studies on amino acid metabolism and its application for industrial and medical purposes.
\end{abstract}

Keywords: Homoserine dehydrogenase, Bacillus subtilis

Received: May 1, 2020 Accepted: September 25, 2020

First published online: September 28, 2020

*Corresponding author Phone: +82-2-820-0433 Fax: +82-2-824-4383 E-mail: jinkukyang@ssu.ac.kr ${ }^{\dagger}$ These authors contributed equally to this work.

pISSN 1017-7825 eISSN 1738-8872

Copyright(C) 2020 by The Korean Society for Microbiology and Biotechnology

\section{Introduction}

Homoserine dehydrogenase (HSD; E.C. 1.1.1.3) catalyzes the reversible conversion of L-aspartate- $\beta$ semialdehyde (L-ASA) to L-homoserine (L-HSE) (Fig. 1). The oxidation of L-ASA to L-HSE is an important step in the aspartate pathway involved in the biosynthesis of L-threonine, L-methionine, and L-isoleucine [1]. L-ASA is the precursor for the synthesis of L-lysine via an alternative branch of the aspartate pathway. Thus, for large-scale industrial production of L-lysine, proper control of HSD is critical in improving productivity [2]. In addition to its industrial application, HSD attracts great medical interest because the aspartate pathway is present only in plants and microorganisms, not in mammals, and furthermore, HSD is a major regulatory enzyme in the pathway. This suggests that HSD is an attractive target for the development of new pesticides and antibiotics with minimal side effects in humans [2]. In this regard, the antifungal natural product (S)-2-amino-4-oxo-5-hydroxylpentanoic acid has been demonstrated to directly target HSD [1].

To date, structural and enzymatic characterization studies of HSDs have been reported for several microorganisms [2-6]. These studies have revealed features including the dimeric assembly, overall structure, catalytic key residues, and possible reaction mechanism. In particular, the dimeric assembly is a common feature for all the previously reported HSDs. For example, HSD from Staphylococcus aureus forms a dimer in solution as shown by size-exclusion chromatography analysis [5], and the dimeric assembly was confirmed in the crystal structure analysis. HSDs from Saccharomyces cerevisiae, Thermus thermophilus, Pyrococcus horikoshii, and Sulfolobus tokodaii also form dimers in the same manner in the crystal structure lattice [2-4, 6]. All of these HSDs commonly consist of two domains: a nucleotide binding domain and a substrate binding domain. In these structures, the HSDs have a conserved dimeric interface contributed by both domains.

HSD from Bacillus subtilis (BsHSD) has 433 amino acid residues and includes a C-terminal ACT domain as a third domain which is not present in the above previously reported two-domain HSDs. More interestingly, we observed that BsHSD exists as a tetramer in contrast to the canonical two-domain HSDs forming dimers. Thus, to form the tetramer, BsHSD may need another type of dimeric interaction in addition to the previously 


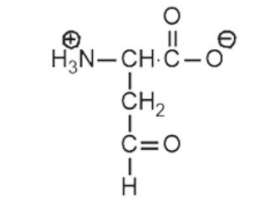

L-aspartate semialdehyde

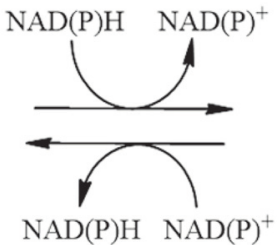

Homoserine dehydrogenase

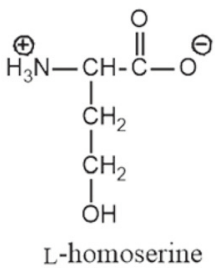

L-homoserine

Fig. 1. Reaction catalyzed by homoserine dehydrogenase (HSD). HSD catalyzes the reversible conversion between L-aspartate semialdehyde and L-homoserine with a cofactor $\mathrm{NAD}(\mathrm{P})^{+}$in oxidation and or $\mathrm{NAD}(\mathrm{P}) \mathrm{H}$ in reduction.

characterized conserved one. Notably, the ACT domain found in many other ACT domain-containing enzymes can homo-dimerize as shown in phosphoglycerate dehydrogenase (PGDH), aspartate kinase (AK), acetohydroxyacid synthase (AHAS) and threonine deaminase (TD), which are all involved in amino acid biosynthesis processes [7-12]. In the crystal structures of these ACT domain-containing enzymes, 2 ACT domains forms a single eight-stranded sheet from the 2 four-stranded $\beta$-sheets. Thus, BsHSD represents a new class of HSD with features of the three-domain architecture and the tetramer formation. In this study, we investigated this novel BsHSD for its molecular and enzymatic features. We overexpressed the recombinant BsHSD in an E. coli system, and purified it to homogeneity. Then, we carried out the enzyme assay and determined the kinetic parameters for L-HSE, the substrate, and for $\mathrm{NADP}^{+}$, the specific cofactor. In addition, we also performed several molecular analyses including size-exclusion chromatography, circular dichroism spectroscopy, and thermal denaturation. In particular, the size-exclusion chromatography revealed that BsHSD forms a tetramer in solution. Finally, we built a homology model for its three-dimensional structure which showed the three-domain architecture of BsHSD, in contrast with the previously reported two-domain dimeric HSDs from other species [2-6]. Here, we report the detailed results from our investigations on the molecular and enzymatic features of BsHSD.

\section{Materials and Methods}

\section{Construction of Expression Plasmid}

The BsHSD-encoding gene, hom, was amplified using the genomic DNA of the B. subtilis strain 168 as a template. The following primers were used for its amplification.

$5^{\prime}$-agatatacatatgaaagcgattcgtgta- $3^{`}$ (forward)

$5^{\prime}$-ctgttctcgaggctccaaccgttcccttc-3'(reverse)

The underlined parts of the sequences indicate NdeI and Xhol restriction enzyme sites. The expression vector, pBsHSD-26b, encoding BsHSD with an additional hexahistidine tag at its C-terminus was constructed by ligation of the digested PCR product into pET26b (Novagen, USA). The sequence of the inserted gene was confirmed by nucleotide sequencing analysis (Bionics, Korea). All reagents were purchased from Sigma-Aldrich (USA) and BioShop (Canada). Genomic DNA of B. subtilis ATCC 23857 was purchased from American Type Culture Collection (ATCC, USA). PCR kits and Mini Plasmid kits from GeneAll (Korea) were used for DNA purification and plasmid isolation. DNA information was retrieved from the NCBI GenBank database (https:// www.ncbi.nlm.nih.gov/gnebank/). The gene ID of hom reported in this paper is CAB15216.1, and the genome accession number of B. subtilis strain 168 is AL009126. The protein information was retrieved from the UniProt database (https://www.uniprot.org) with the accession number P19582.

\section{Protein Overexpression and Purification}

The expression vector, pBsHSD-26b, was transformed into Rosetta2 (DE3) strain of E. coli for overexpression. The cells were cultured in $\mathrm{LB}$ media at $37^{\circ} \mathrm{C}$ with shaking until the $\mathrm{OD}_{600}$ reached $0.5-0.8$. Then, the culturing flask was cooled in an ice bath for about $10 \mathrm{~min}$ and isopropyl- $\beta$-D-1-thiogalactopyranoside (IPTG) was added to a final concentration of $0.5 \mathrm{mM}$ to induce BsHSD expression. The cells were cultured again for an additional $20 \mathrm{~h}$ at $25^{\circ} \mathrm{C}$. The cultured cells were harvested by centrifugation at 1,590 $\mathrm{g}$ for $20 \mathrm{~min}$ (Hanil Supra 22K), and then the cell pellets were resuspended in lysis buffer containing $20 \mathrm{mM}$ Tris- $\mathrm{HCl} \mathrm{pH} 8.0,0.4 \mathrm{M} \mathrm{NaCl}, 0.1 \mathrm{mM}$ TCEP, $5 \%$ glycerol, $10 \mathrm{mM}$ imidazole and $0.1 \mathrm{mM}$ phenyl methyl sulfonyl fluoride (PMSF). The resuspended cells were lysed by sonification and then centrifuged at 24,650 $\mathrm{g}$ for $60 \mathrm{~min}$ (Hanil Supra 22K). Next, the BsHSD was purified from the supernatants through 3 serial applications of column chromatography using a HisTrapFF, HiPrep 26/10 Desalting and Superdex-200 (GE Healthcare, USA). The protein purity was examined with Sodium Dodecyl Sulfate-Polyacrylamide Gel Electrophoresis (SDS-PAGE), and the concentration was measured using a NanoDrop 1000 (Thermo Scientific, USA).

\section{Activity Assay and Kinetic Analysis}

The homoserine oxidation was monitored at $25^{\circ} \mathrm{C}$ for all enzyme assays. The absorbance at $340 \mathrm{~nm}$ was monitored using an Ultrospec $8000 \mathrm{UV} /$ Vis spectrophotometer (GE Healthcare) and the concentration of NADPH was calculated from its extinction coefficient at $6.22 \mathrm{mM}^{-1} \mathrm{~cm}^{-1}$. To determine the optimal $\mathrm{NaCl}$ concentration, 6 varying $\mathrm{NaCl}$ concentrations $(50,100,200,400,800$, and 1,600 $\mathrm{mM}$ ) were examined in the presence of $100 \mathrm{mM}$ CHES $\mathrm{pH}$ 9.5, $50 \mathrm{mM} \mathrm{L-HSE}, 0.5 \mathrm{mM} \mathrm{NADP}^{+}$and $1 \mu \mathrm{M}$ BsHSD. To screen for the optimum 
$\mathrm{pH}$ range, three different buffer systems were used to cover the inspected $\mathrm{pH}$ range of 7.0 to 10.0: Bis-Tris buffer for $\mathrm{pH} 7.0$, Tris-HCl buffer for $\mathrm{pH} 8.0$ and 9.0, CAPS buffer for $\mathrm{pH} 10.0$ and 11.0. The reactions at different $\mathrm{pHs}$ were monitored under the standard conditions of $100 \mathrm{mM} \mathrm{L-HSE}, 1 \mathrm{mM} \mathrm{NADP}^{+}, 400 \mathrm{mM} \mathrm{NaCl}$ and $0.5 \mu \mathrm{MBsHSD}$. To investigate the temperature dependence of the enzyme activity, the enzyme activity was measured at various temperatures from $25^{\circ} \mathrm{C}$ to $50^{\circ} \mathrm{C}$ with increments of $5^{\circ} \mathrm{C}$ in a reaction mixture containing $100 \mathrm{mM} \mathrm{L}-\mathrm{HSE}, 1 \mathrm{mM}$ $\mathrm{NADP}^{+}, 400 \mathrm{mM} \mathrm{NaCl}, 0.5 \mu \mathrm{M}$ BsHSD and $100 \mathrm{mM}$ CHES buffer, at pH 9.0. Subsequent kinetic analyses were carried out under the determined optimum condition for $\mathrm{NaCl}$ concentration and $\mathrm{pH}(400 \mathrm{mM} \mathrm{NaCl}$ and $\mathrm{pH} 9.0)$ for $0.5 \mu \mathrm{M}$ BsHSD. The cofactor preference between $2 \mathrm{mM} \mathrm{NAD}^{+}$and $1 \mathrm{mM} \mathrm{NADP}^{+}$was investigated with $100 \mathrm{mM} \mathrm{L}-\mathrm{HSE}$ and $0.5 \mu \mathrm{M}$ BsHSD to determine the kinetic parameters for substrate and cofactor. The initial reaction rate was measured for L-HSE and $\mathrm{NADP}^{+}$at 6,7 different concentrations, ranging from $1 \mathrm{mM}$ to $300 \mathrm{mM}$ and $20 \mathrm{mM}$ to $2000 \mathrm{mM}$, respectively. The concentration of L-HSE was varied with NADP ${ }^{+}$concentration fixed at $2 \mathrm{mM}$, or the concentration of $\mathrm{NADP}^{+}$was varied with L-HSE concentration fixed at $100 \mathrm{mM}$. Freshly purified protein samples were used for all enzyme assays and the data from 3 independent experiments were averaged for one measurement value.

All apparent kinetic parameters were calculated with Origin 9.0 software (OriginLab, USA). The MichaelisMenten equation $V=V_{\max } \mathrm{S} /\left(K_{m}+\mathrm{S}\right)$ was used as the reference equation to calculate the apparent parameters from the optimal fitting.

\section{Size-Exclusion Chromatography Analysis}

Size-exclusion chromatography was carried out using a Superdex-200 10/300 GL column on an AKTA basic system (GE Healthcare). A $200 \mu \mathrm{l}$ protein sample was injected into the column, which was pre-equilibrated with working buffer $(20 \mathrm{mM}$ Tris- $\mathrm{HCl}, 0.1 \mathrm{mM} \mathrm{TCEP}$, and $400 \mathrm{mM} \mathrm{NaCl}, \mathrm{pH} 8.0)$. The injected protein sample was run through the column at a flow rate of $0.5 \mathrm{~mL} / \mathrm{min}$. To determine the void volume, Blue dextran (Sigma-Aldrich, D4772) was used. Next, the mixture of the other 8 standard proteins was run: aprotinin from bovine lung (SigmaAldrich, A3886), cytochrome C from equine heart (Sigma-Aldrich, C7150), carbonic anhydrase from bovine erythrocytes (Sigma-Aldrich, C7025), ovalbumin from chicken egg white (Sigma-Aldrich, A8581), alcohol dehydrogenase from yeast (Sigma-Aldrich, A8656), $\beta$-amylase from sweet potato (Sigma-Aldrich, A8781), and apoferritin from horse spleen (Sigma-Aldrich, A3630). From all the above elution volume measurements, the standard curve for the molecular mass was established. The elution volume of BsHSD was also measured under conditions similar to those used for the standard proteins.

\section{Circular Dichroism (CD) Spectroscopy and Thermal Denaturation Analysis}

Prior to the CD measurements, the buffer condition for the BsHSD sample was changed $(20 \mathrm{mM}$ Tris- $\mathrm{HCl} \mathrm{pH}$ 8.0, $400 \mathrm{mM} \mathrm{NaF}$, and 0.1 mM TCEP) and a HiPrep 26/10 Desalting column was used. The far-ultraviolet CD spectrum of BsHSD was recorded at $20^{\circ} \mathrm{C}$ within a cell with $0.1 \mathrm{~cm}$ path length using a Jasco J-710 spectropolarimeter (JASCO, USA). Three individual scans were recorded from $190 \mathrm{~nm}$ to $260 \mathrm{~nm}(0.1 \mathrm{~nm}$ step resolution, $1 \mathrm{~nm}$ bandwidth and $1 \mathrm{~s}$ response time). The solvent CD signal was subtracted after summing and averaging 3 spectra. The CD intensity was normalized to the mean residue molar ellipticity. The thermal denaturation experiment was carried out at $222 \mathrm{~nm}$ for BsHSD at a concentration of $0.5 \mathrm{mg} / \mathrm{ml}$. The CD intensity was recorded every $30 \mathrm{~s}$ as the temperature increased from $26^{\circ} \mathrm{C}$ to $94^{\circ} \mathrm{C}$ at a speed of $2^{\circ} \mathrm{C} / \mathrm{min}$.

\section{Homology Modeling}

The three-dimensional structure was predicted by SWISS-MODEL (http://swissmodel.expasy.org) from the amino acid sequence of BsHSD. The server selected the crystal structure of HSD from Mycolicibacterium hassiacum (PDB - 6DZS; not published) as a template, on which the structure of BsHSD was modeled.

\section{Results \\ BsHSD Expression and Purification}

Expression of the recombinant BsHSD in E. coli at $37^{\circ} \mathrm{C}$ resulted mostly into inclusion bodies. However, we found that it can be expressed in soluble form at a lower temperature of $25^{\circ} \mathrm{C}$. The band for expressed BsHSD appeared between two markers, $48.5 \mathrm{kDa}$ to $42.8 \mathrm{kDa}$, which is in good agreement with its calculated molecular mass of $48.3 \mathrm{kDa}$. The expressed recombinant BsHSD was purified through a series of chromatography columns. The final purified sample showed remarkably high homogeneity, which was assessed with SDS-PAGE (Fig. 2), and it was applied to the subsequent molecular and enzymatic analyses.

\section{Optimal Enzymatic Condition and Cofactor Specificity}

To establish the optimal salt concentration and $\mathrm{pH}$ for the L-HSE oxidation reaction catalyzed by BsHSD, we measured the catalytic activity at varied $\mathrm{NaCl}$ concentrations and $\mathrm{pH}$, respectively. First, we tested 7 different $\mathrm{NaCl}$ concentrations ranging from 0 to $1.6 \mathrm{M}$ at $\mathrm{pH} 9.5$ (Fig. $3 \mathrm{~A}$ ). The results clearly showed that the presence of $\mathrm{NaCl}$ is greatly beneficial for the enzyme activity and even with only $50 \mathrm{mM} \mathrm{NaCl}$, the activity was about 11 times greater than the activity without $\mathrm{NaCl}$. As the $\mathrm{NaCl}$ concentration was doubled from $50 \mathrm{mM}$ to $100 \mathrm{mM}$, and then sequentially to $1,600 \mathrm{mM}$, the activity increased to reach its maximum at $400 \mathrm{mM} \mathrm{NaCl}$ with a specific activity of $1.48 \pm 0.12 \mu \mathrm{mol} \cdot \mathrm{min}^{-1} \cdot \mathrm{mg}^{-1}$. Thus, all the subsequent assays were carried out with the $100 \mathrm{mM}$ Tris- $\mathrm{HCl}$ buffer containing $400 \mathrm{mM} \mathrm{NaCl}$. Next, we investigated the $\mathrm{pH}$ dependence of L-HSE oxidation by measuring the activity at 5 different $\mathrm{pHs}(7.0,8.0,9.0,10.0$, and 11.0) (Fig. 3B). BsHSD was maximally active for L-HSE oxidation at $\mathrm{pH}$ 9.0 with a specific activity of $2.01 \pm 0.01 \mu \mathrm{mol} \cdot \mathrm{min}^{-1} \cdot \mathrm{mg}^{-1}$ and was the least active at $\mathrm{pH} 7.0$ with only $1.1 \%$ of the 


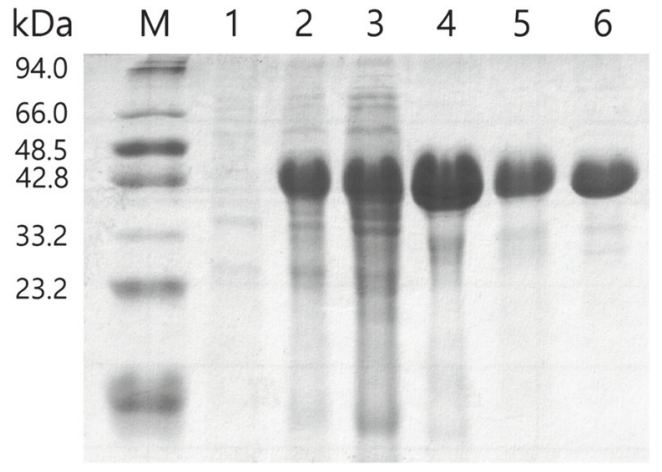

Fig. 2. Purification of recombinant BsHSD. SDS-PAGE for the samplings in the steps of culturing and purification. Lane M: protein marker; Lane 1: before IPTG addition; Lane 2: overnight, after IPTG addition; Lane 3: supernatant after cell lysis; Lane 4: elution from HistrapFF column; Lane5: after Superdex-200 column; and Lane 6: final concentrated sample.
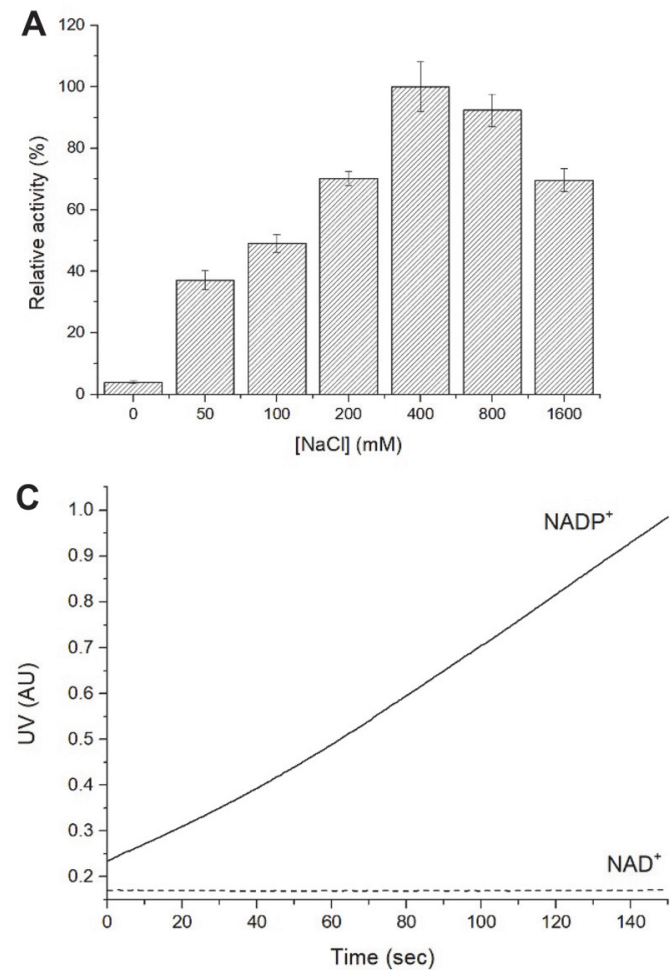

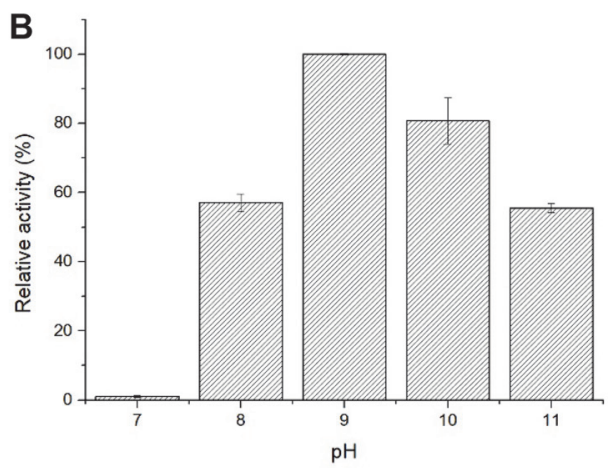

D

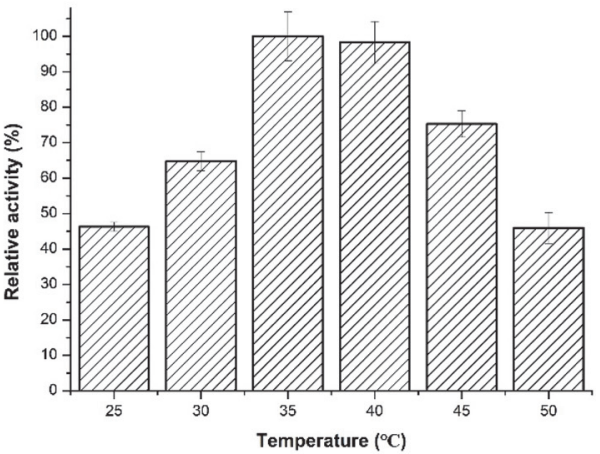

Fig. 3. Optimum conditions for BsHSD. The specific activity was measured at varying points of parameters to inspect the optimum condition. (A) Effect of $\mathrm{NaCl}$ concentration (B) $\mathrm{pH}$ dependence (C) Cofactor specificity (D) Temperature dependence of the enzymatic activity.

maximum activity. We also examined the cofactor specificity of L-HSE oxidation for $\mathrm{NAD}^{+}$and $\mathrm{NADP}^{+}$. BsHSD exhibited absolute preference for $\mathrm{NADP}^{+}$and the activity with $\mathrm{NAD}^{+}$was not detectable at all (Fig. $3 \mathrm{C}$ ). We also carried out the homoserine oxidation assay at different reaction temperatures ranging from $25^{\circ} \mathrm{C}$ to $50^{\circ} \mathrm{C}$ to investigate the temperature dependence of the enzyme activity. As the temperature increased, the specific activity also increased to $35^{\circ} \mathrm{C}$ and then gradually decreased to $40^{\circ} \mathrm{C}$ (Fig. 3D).

\section{Apparent Kinetic Parameters}

To determine the kinetic parameters for the oxidation of L-homoserine by BsHSD, we measured the initial reaction rates at various concentrations of L-homoserine with $\mathrm{NADP}^{+}$saturation or conversely at various concentrations of $\mathrm{NADP}^{+}$with L-homoserine saturation (Fig. 4). The $K_{\mathrm{m}}$ values for L-homoserine and NADP ${ }^{+}$ were $35.08 \pm 2.91 \mathrm{mM}$ and $0.39 \pm 0.05 \mathrm{mM}$ (Table 1), respectively, and the $V_{\max }$ values were quite similar at $2.72 \pm$ $0.06 \mu \mathrm{mol} \cdot \mathrm{min}^{-1} \cdot \mathrm{mg}^{-1}$ and $2.79 \pm 0.11 \mu \mathrm{mol} \cdot \mathrm{min}^{-1} \cdot \mathrm{mg}^{-1}$ respectively, with the $k_{\text {cat }} / K_{\mathrm{m}}$ value as $0.03 \pm 0.01 \mathrm{sec}^{-1} \mathrm{mM}^{-1}$, indicating that the binding of $\mathrm{L}$-homoserine is the rate limiting factor for L-homoserine oxidation. 

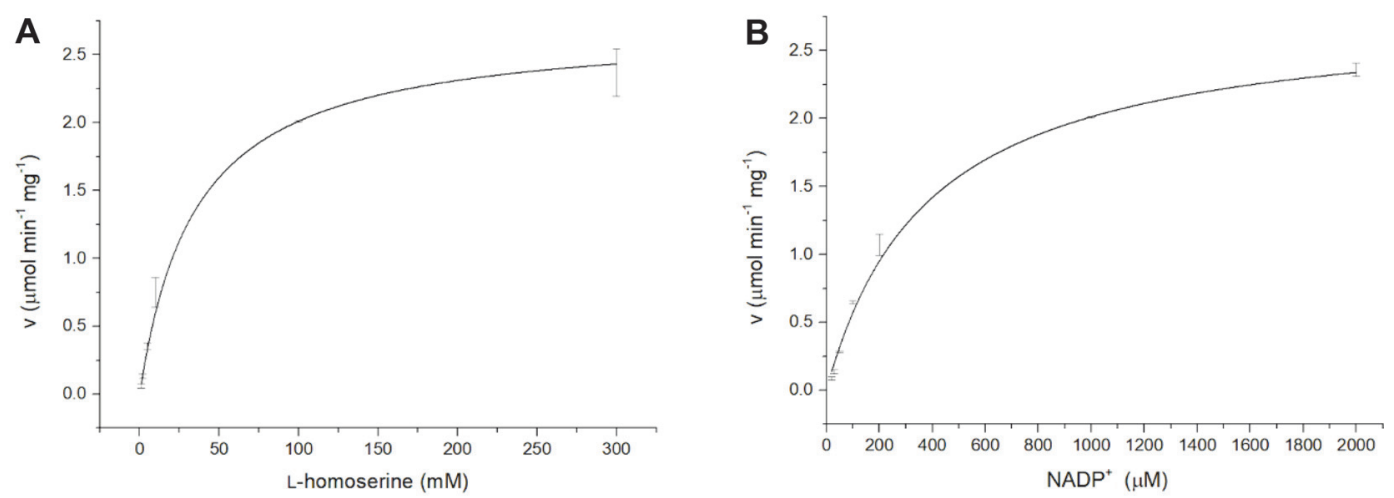

Fig. 4. Michaelis-Menten kinetics of BsHSD. The specific activity was measured at varying concentration points of substrate or cofactor to determine the kinetic parameters. (A) L-homoserine concentration was varied with saturated concentration of $\mathrm{NADP}^{+}$. (B) $\mathrm{NADP}^{+}$concentration was varied with saturated concentration of L-homoserine.

Table 1. Kinetic parameters.

\begin{tabular}{lcccc}
\hline \multicolumn{1}{c}{ Substrate } & $\begin{array}{c}V_{\max } \\
\left(\mu \mathrm{mol} \mathrm{min} \mathrm{mg}^{-1}\right)\end{array}$ & $\begin{array}{c}K_{\mathrm{m}} \\
(\mathrm{mM})\end{array}$ & $\begin{array}{c}k_{\text {cat }} \\
\left(\mathrm{sec}^{-1}\right)\end{array}$ & $\begin{array}{c}k_{\text {cat }} / K_{\mathrm{m}} \\
\left(\mathrm{sec}^{-1} \mathrm{mM}^{-1}\right)\end{array}$ \\
\hline L-homoserine & $2.72 \pm 0.06$ & $35.08 \pm 2.91$ & $1.07 \pm 0.02$ & $0.03 \pm 0.01$ \\
$\mathrm{NADP}^{+}$ & $2.79 \pm 0.11$ & $0.39 \pm 0.05$ & $1.10 \pm 0.04$ & $2.86 \pm 0.91$ \\
\hline
\end{tabular}

\section{Size-Exclusion Chromatography Analysis}

To examine the oligomeric states of BsHSD, we carried out size-exclusion chromatography. The molecular mass was estimated by comparing the elution volume of BsHSD with those of the standard proteins (Fig. 5). The BsHSD peak emerged at $12.0 \mathrm{ml}$ of elution volume and the molecular mass was calculated to be $221 \mathrm{kDa}$ from the standard curve, which was 4.6 times greater than the actual molecular mass of monomeric BsHSD (48.3 kDa). This result indicates that BsHSD exists as a tetramer in solution.

\section{Circular Dichroism Spectroscopy and Thermal Denaturation}

The CD spectra were recorded for the wavelength scan from $190 \mathrm{~nm}$ to $260 \mathrm{~nm}$ at $25^{\circ} \mathrm{C}$ (Fig. 6A), and it exhibited a typical pattern for $\alpha / \beta$ proteins. The content of the secondary structure elements was estimated by BeStSel [13], and it showed $16.7 \%$ for helices, $25.0 \%$ for strands, $14.3 \%$ for turns, and $43.9 \%$ for the others. This is largely consistent with the previous reports of the crystal structures of HSDs from other organisms which revealed a common $\alpha / \beta$ structure including a Rossman fold [2-6]. In addition, this result suggests the structural integrity of the purified BsHSD sample. Next, thermal denaturation experiments were performed using CD spectroscopy to investigate the thermal stability of BsHSD. The spectrum was measured every $30 \mathrm{~s}$ at $222 \mathrm{~nm}$, raising the sample temperature from $26^{\circ} \mathrm{C}$ to $94^{\circ} \mathrm{C}$ and a total of 336 data points were collected. The melting temperature (Tm) was determined from the sigmoid fitting of these data at $54.8 \pm 0.1^{\circ} \mathrm{C}$, which fell within the range for mesophilic proteins $\left(20^{\circ} \mathrm{C}\right.$ to $\left.60^{\circ} \mathrm{C}\right)[14,15]$.

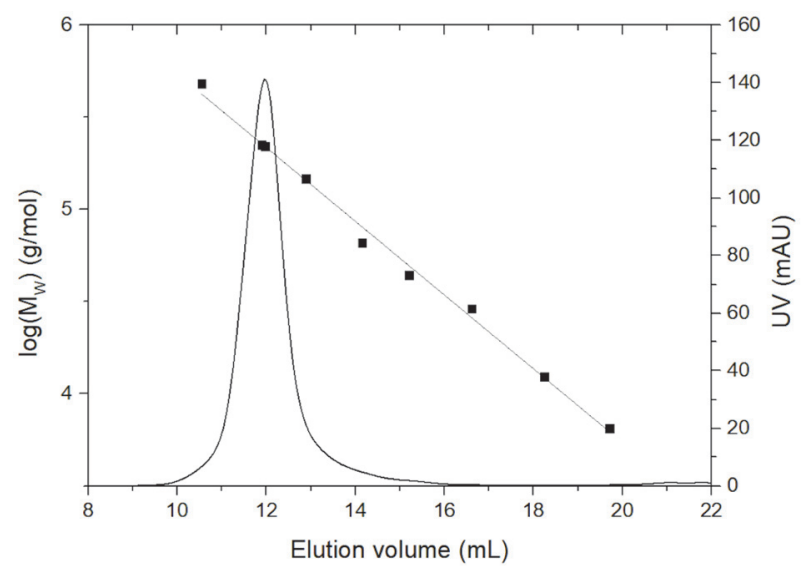

Fig. 5. Size-exclusion chromatography analysis. The molecular mass of BsHSD was measured in size-exclusion column chromatography to investigate the oligomeric state in solution. Filled squares are 8 different standard proteins. The peak corresponds to BsHSD eluted at $12.0 \mathrm{ml}$. 
A

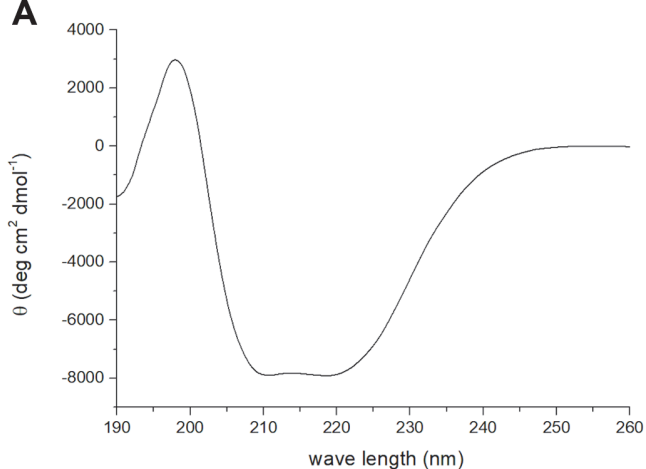

B

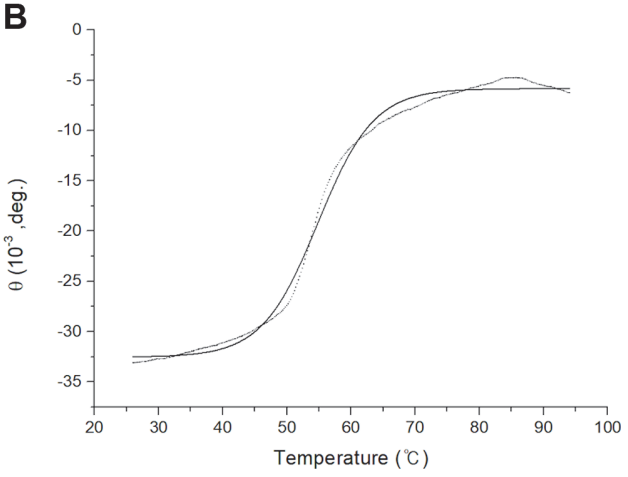

Fig. 6. Circular dichroism analysis. The fold integrity and the secondary structure composition was checked from the wavelength scan, and the thermal denaturation experiment was carried out to investigate the thermal stability. (A) Wavelength scan (B) Thermal denaturation at $222 \mathrm{~nm}$. The dotted line represents raw measurements, and the solid line represents their sigmoidal fitting.

\section{Predicted Model for Three-Dimensional Structure}

The three-dimensional structure of BsHSD was predicted using the SWISS-MODEL server (http:// swissmodel.expasy.org) based on the crystal structure of HSD from M. hassiacum (PDB - 6DZS; MhHSD, hereafter; not published) as a homology model that was algorithmically selected by the server. MhHSD showed $40.3 \%$ sequence identity with BsHSD on an alignment covering 430 residues with several short gaps. The model revealed that BsHSD consists of 3 domains: the $\mathrm{N}$-terminal nucleotide-binding domain of a varied Rossman fold, a central substrate-binding domain, and a C-terminal ACT domain (acronym for aspartate kinase, chorismate mutase and TyrA (prephenate dehydrogenase)) of a ferredoxin-like fold (Fig. 7) [5]. The nucleotide-binding domain and the substrate-binding domain are commonly found in all HSDs from any organism, but the Cterminal ACT domain is an additional regulatory domain that is present in only a subset of HSDs [2-6].

\section{Discussion}

In this study, BsHSD, was recombinantly expressed, purified and characterized for its molecular and enzymatic features. We investigated the optimal $\mathrm{pH}$, temperature, and $\mathrm{NaCl}$ concentration for the enzyme activity and determined the kinetic parameters for the oxidation of L-homoserine. Next, we also examined the oligomeric state of BsHSD in solution through size-exclusion chromatography, and its thermal stability by CD spectroscopic thermal denaturation test. Finally, we built a predicted model for three-dimensional structure of BsHSD. Based on all these analyses, a few novel features of BsHSD were revealed. In the preliminary enzyme assays for the optimization of the reaction conditions, we observed that the enzyme activity was greatly enhanced (almost 11

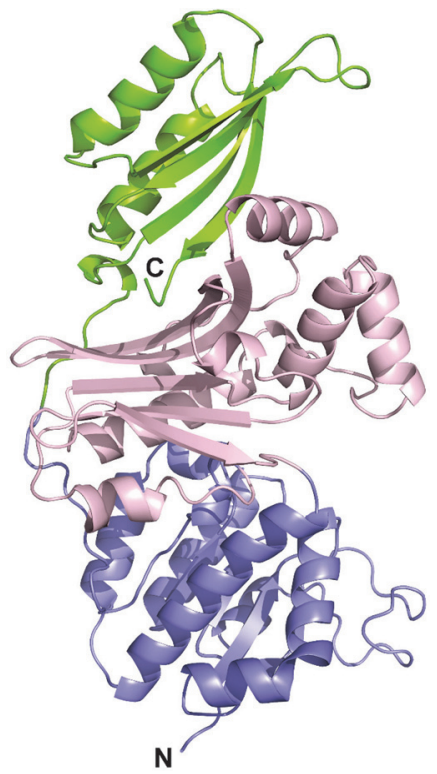

Fig. 7. Predicted model for three-dimensional structure of BsHSD. The N-terminal nucleotide-binding domain is shown in blue, the central substrate-binding domain in pink, and the C-terminal ACT domain in green. 
times) by the addition of $\mathrm{NaCl}$ even at very low concentration $(50 \mathrm{mM})$ and the activity reached its maximum at $400 \mathrm{mM}$ of $\mathrm{NaCl}$ concentration. In this regard, it should be noted that BaHSD showed the tendency to aggregate over time, as assessed by the appearance of the peak at void volume in the absence of $\mathrm{NaCl}$ and the removal of this aggregation almost completely by the addition of $400 \mathrm{mM} \mathrm{NaCl}$, as shown by the size-exclusion chromatography data. Therefore, it seems that the activity enhancement by $\mathrm{NaCl}$ may have resulted at least in part because of preventing aggregation.

Since the dimer is a typical oligomeric state for the previously reported HSDs from other organisms [2-6], the tetramer formation of BsHSD was an unexpected observation. For example, HSD from S. aureus was shown to exist as a dimer in solution as examined by size-exclusion chromatography [5], which was consistent with its crystal structure revealing the dimeric assembly. Moreover, HSDs from S. cerevisiae, T. thermophilus, P. horikoshii, and $S$. tokodaii also showed the same dimeric assembly in the crystal lattice [2-4,6], even though their oligomeric state was not investigated in solution. These previous crystal structures of HSDs commonly showed that the dimeric interface was contributed by both the substrate-binding domain and the nucleotide-binding domain. Thus, to form the tetramer, BsHSD needs another type of dimeric interaction in addition to the above conserved one. In this regard, it is noteworthy that BsHSD contains the additional C-terminal ACT domain, which is not present in all of the above dimeric HSDs, with the exception of HSD from S. aureus. More importantly, the ACT domain was shown to be responsible for the dimerization of the many other ACT domain-containing enzymes such as PGDH, AK, AHAS, and TD, which are all HSDs involved in amino acid biosynthesis processes [7-12]. An ACT domain adopts a ferredoxin-like fold of $\beta \alpha \beta \beta \alpha \beta$ which has a four-stranded $\beta$-sheet with $2 \alpha$-helices flanked on one side. In the crystal structures of these ACT domain-containing enzymes, 2 ACT domains accomplish dimerization in a conserved way with 2 four-stranded $\beta$-sheets joined edge-to-edge into a single eight-stranded sheet. Therefore, it is very tempting to speculate that the additional dimeric interface responsible for the tetramer formation of BsHSD must be established by its C-terminal ACT domain. Subsequent structural analysis will clearly reveal the details of the novel tetrameric assembly of BsHSD.

Conclusively, the molecular and biochemical features of BsHSD were investigated in this study. Given the importance of HSD as a key enzyme in the aspartate pathway and also as a target for medical and industrial applications, this study will add to the knowledge on the biochemistry of amino acid metabolism and lay the foundation for future efforts in corresponding applications.

\section{Acknowledgments}

This research was supported by the Basic Science Research Program of the National Research Foundation of Korea (NRF), funded by the Ministry of Science, ICT and Future Planning (NRF-2017R1D1A1B03035446).

\section{Conflict of Interest}

The authors have no financial conflicts of interest to declare.

\section{References}

1. Jacques SL, Nieman C, Bareich D, Broadhead G, Kinach R, Honek JF, et al. 2001. Characterization of yeast homoserine dehydrogenase, an antifungal target: the invariant histidine 309 is important for enzyme integrity. Biochim. Biophys. Acta 1544: 28-41.

2. Akai S, Ikushiro H, Sawai T, Yano T, Kamiya N, Miyahara I. 2019. The crystal structure of homoserine dehydrogenase complexed with l-homoserine and NADPH in a closed form. J. Biochem. 165: 185-195.

3. DeLaBarre B, Thompson PR, Wright GD, Berghuis AM. 2000. Crystal structures of homoserine dehydrogenase suggest a novel catalytic mechanism for oxidoreductases. Nat. Struct. Biol. 7: 238-244.

4. Hayashi J, Inoue S, Kim K, Yoneda K, Kawarabayasi Y, Ohshima T, et al. 2015. Crystal structures of a hyperthermophilic archaeal homoserine dehydrogenase suggest a novel cofactor binding mode for oxidoreductases. Sci. Rep. 5: 11674 .

5. Navratna V, Reddy G, Gopal B. 2015. Structural basis for the catalytic mechanism of homoserine dehydrogenase. Acta Crystallogr. D Biol. Crystallogr. 71: 1216-1225.

6. Tomonaga Y, Kaneko R, Goto M, Ohshima T, Yoshimune K. 2015. Structural insight into activation of homoserine dehydrogenase from the archaeon Sulfolobus tokodaii via reduction. Biochem. Biophys. Rep. 3: 14-17.

7. Curien G, Biou V, Mas-Droux C, Robert-Genthon M, Ferrer JL, Dumas R. 2008. Amino acid biosynthesis: new architectures in allosteric enzymes. Plant Physiol. Biochem. 46: 325-339.

8. Schuller DJ, Grant GA, Banaszak LJ. 1995. The allosteric ligand site in the Vmax-type cooperative enzyme phosphoglycerate dehydrogenase. Nat. Struct. Biol. 2: 69-76.

9. Mas-Droux C, Curien G, Robert-Genthon M, Laurencin M, Ferrer JL, Dumas R. 2006. A novel organization of ACT domains in allosteric enzymes revealed by the crystal structure of Arabidopsis aspartate kinase. Plant Cell 18: 1681-1692.

10. Yoshida A, Tomita T, Kurihara T, Fushinobu S, Kuzuyama T, Nishiyama M. 2007. Structural Insight into concerted inhibition of alpha 2 beta 2-type aspartate kinase from Corynebacterium glutamicum. J. Mol. Biol. 368: 521-536.

11. Kaplun A, Vyazmensky M, Zherdev Y, Belenky I, Slutzker A, Mendel S, et al. 2006. Structure of the regulatory subunit of acetohydroxyacid synthase isozyme III from Escherichia coli. J. Mol. Biol. 357: 951-963.

12. Gallagher DT, Gilliland GL, Xiao G, Zondlo J, Fisher KE, Chinchilla D, et al. 1998. Structure and control of pyridoxal phosphate dependent allosteric threonine deaminase. Structure 6: 465-475.

13. Micsonai A, Wien F, Kernya L, Lee YH, Goto Y, Refregiers M, et al. 2015. Accurate secondary structure prediction and fold recognition for circular dichroism spectroscopy. Proc. Natl. Acad. Sci. USA 112: E3095-E3103.

14. Stover CK, Pham XQ, Erwin AL, Mizoguchi SD, Warrener P, Hickey MJ, et al. 2000. Complete genome sequence of Pseudomonas aeruginosa $\mathrm{PAO} 1$, an opportunistic pathogen. Nature 406: 959-964.

15. Pucci F, Rooman M. 2017. Physical and molecular bases of protein thermal stability and cold adaptation. Curr. Opin. Struct. Biol. 42: $117-128$. 